Publ. Mat. 63 (2019), 265-293

DOI: 10.5565 /PUBLMAT6311909

\title{
SIMPLICIAL LUSTERNIK-SCHNIRELMANN CATEGORY
}

\author{
Desamparados Fernández-Ternero, Enrique Macías-Virgós, \\ Erica Minuz, and José Antonio Vilches
}

\begin{abstract}
The simplicial LS-category of a finite abstract simplicial complex is a new invariant of the strong homotopy type, defined in purely combinatorial terms. We prove that it generalizes to arbitrary simplicial complexes the well known notion of arboricity of a graph, and that it allows to develop many notions and results of algebraic topology which are costumary in the classical theory of Lusternik-Schnirelmann category. Also we compare the simplicial category of a complex with the LS-category of its geometric realization and we discuss the simplicial analogue of the Whitehead formulation of the LS-category.
\end{abstract}

2010 Mathematics Subject Classification: 55U10, 55M30, 06F30.

Key words: Lusternik-Schnirelmann category, strong homotopy type, geometric realization, Whitehead formulation of category, graph arboricity.

\section{Introduction}

In a previous paper $[\mathbf{9}]$ we introduced the so-called simplicial Lusternik-Schnirelmann category of a simplicial complex. This invariant, denoted by scat $K$, is defined directly from the combinatorial structure of the complex $K$, instead of considering the topological LS-category of the geometric realization $|K|$, by replacing the notion of homotopy by that of contiguity. With this approach it turned out that the simplicial LS-category is an invariant of the strong homotopy type, as defined by Barmak and Minian in [4]. Also, simplicial LS-category is closely related to the LS-category of the finite $T_{0}$-space represented by the Hasse diagram of $K[\mathbf{1 8}]$.

It is worth noting that although the idea of contiguous simplicial maps is a classic one, the corresponding theory of Lusternik-Schnirelmann category had not been developed until now. Then, many natural questions remained unsolved in this new context. The aim of this paper is to answer some of them: for instance, the relationship between scat $K$ and cat $|K|$ (Theorem 4.4) or the Whitehead formulation of simplicial category (Definition 6.5), among others. Other results include a direct proof 
of the formula scat $(\operatorname{sd} K) \leq$ scat $K$ for the barycentric subdivision (Theorem 3.1), or a counterexample of the analogue of the homotopy extension property for subcomplexes (Example 7.6). A final important result (Theorem 8.12) will be that simplicial category generalizes to arbitrary simplicial complexes the well known notion of arboricity of a graph.

The contents of the paper are as follows.

We start (Section 2) by introducing the definition of simplicial Lusternik-Schnirelmann category scat $K$ of the simplicial complex $K$. The idea is to define an analogue of the classical LS-category of a topological space, by replacing the notion of homotopic maps by that of contiguous simplicial maps. We then prove some basic properties of this new notion of category, and we recall our previous result [9] that scat is an invariant of the strong homotopy type. In particular, the category of the simplicial complex $K$ equals that of its core $K_{0}$ (the minimal subcomplex of $K$ obtained by strong collapses). As a corollary we prove that scat $K$ is bounded above by the number of vertices and the number of maximal simplices of $K_{0}$.

In Section 3 we study the effect of barycentric subdivision on simplicial category and we give a direct proof of $\operatorname{scat}(\operatorname{sd} K) \leq \operatorname{scat} K$. For that we need to prove that if $\varphi, \psi$ are contiguous maps, then the induced maps $\operatorname{sd} \varphi, \operatorname{sd} \psi$, are in the same contiguity class. The only proof that we found in the literature is as a consequence of the results given in [4] via finite posets, so we give a direct one.

In Section 4 we compare the simplicial category of the complex $K$ with the classical LS-category of the geometric realization $|K|$, proving that cat $|K| \leq \operatorname{scat} K$. This implies that cat $|K| \leq \operatorname{scat}\left(\operatorname{sd}^{N} K\right)$, for any iterated barycentric subdivision $\operatorname{sd}^{N} K$ of $K$. We show an example where this inequality is strict.

Section 5 deals with the study of simplicial products. Being more precise, we consider the categorical product, denoted by $K \times L$. Then, we prove that $\operatorname{scat}(K \times L)+1 \leq(\operatorname{scat} K+1)($ scat $L+1)$.

We are now (Section 6) in a position to discuss the simplicial analogue of the Whitehead formulation of the LS-category. It is known that for topological spaces $X$ with "good" properties the LS-category can be computed as the least integer $n=$ cat $^{\text {Wh }} X$ such that the diagonal map $\Delta: X \rightarrow X^{n+1}$ factors (up to homotopy) through the so-called fat wedge $T^{n+1} X$. We try to adapt this result to abstract simplicial complexes. First, we define the $n$-th fat wedge $T^{n} K$ for any pointed complex $K$ and we study its behaviour under contiguity. We then define a simplicial Whitehead category scat ${ }^{\mathrm{Wh}} K$, which is an invariant 
of the pointed strong homotopy type and we are able to prove that scat $K \leq$ scat $^{\mathrm{Wh}} K$. However, unlike the continuous case, the other inequality is not true, as we show by an example.

In Section 7 we explain how the partial failure of the Whitehead formulation is related to the contiguity extension property. We discuss a new notion of cofibration in the simplicial setting. It is well known that if $A$ is a subcomplex of a CW-complex $X$ then the pair $(X, A)$ has the homotopy extension property. Therefore, if $L$ is a subcomplex of a simplicial complex $K$, the pair $(|K|,|L|)$ has the (topological) homotopy extension property. We define a purely combinatorial analogue for a simplicial pair $(K, L)$, but we show counterexamples where the contiguity extension property fails to be true.

Finally, Section 8 is focused on the study of the simplicial LS-category in the one-dimensional case, that is, on graphs. The well known graphtheoretical notion of arboricity will play a central role in this study. The arboricity $\Upsilon(G)$ of the graph $G$ is the cardinality of a minimal decomposition of $G$ into disjoint spanning forests, i.e., acyclic subgraphs which are not necessarily connected and cover all the vertices. The aim of this section is to prove that arboricity coincides with simplicial category. Being more precise, we prove that $\Upsilon(G)=$ scat $G+1$. The two main ideas are that categorical subcomplexes are precisely forests and that the notion of contiguity is a very rigid one in the setting of graphs, allowing only a limited number of moves.

As a final comment, we emphasize that simplicial LS-category is a new strong homotopy invariant, defined in purely combinatorial terms, that generalizes to arbitrary simplicial complexes the well known notion of arboricity of a graph, and that allows to develop all the machinery of algebraic topology which is costumary in the classical theory of Lusternik-Schnirelmann category.

\section{Simplicial category}

2.1. The notion of simplicial LS-category. We recall the notion of simplicial LS-category, introduced by the authors in $[\mathbf{9}]$.

Let $K$ and $L$ be abstract simplicial complexes. Remember (see [20, §3.5]) that two simplicial maps $\varphi, \psi: K \rightarrow L$ are contiguous, denoted by $\varphi \sim_{c} \psi$, if for any simplex $\sigma=\left\{v_{0}, \ldots, v_{p}\right\}$ of $K$, the set of vertices

$$
\varphi(\sigma) \cup \psi(\sigma)=\left\{\varphi\left(v_{0}\right), \ldots, \varphi\left(v_{p}\right), \psi\left(v_{0}\right), \ldots, \psi\left(v_{p}\right)\right\}
$$

is a simplex of $L$. More generally, two simplicial maps $\varphi, \psi: K \rightarrow L$ are in the same contiguity class, denoted by $\varphi \sim \psi$, if there is a sequence of 
simplicial maps $\varphi_{i}: K \rightarrow L, i=1, \ldots, m$, such that $\varphi=\varphi_{1} \sim_{c} \varphi_{2} \sim_{c}$ $\cdots \sim_{c} \varphi_{m}=\psi$.

At this point it is convenient to recall that the subcomplex $L^{\prime} \subseteq L$ is said to be full subcomplex of $L$ if each simplex of $L$ whose vertices belong to $L^{\prime}$ is also a simplex of $L^{\prime}$. The next proposition can be proved easily from this definition.

Proposition 2.1. Let $\varphi, \psi: K \rightarrow L$ be two simplicial maps. Let $K^{\prime} \subset K$ be a subcomplex and let $L^{\prime} \subset L$ be a full subcomplex such that $\varphi\left(K^{\prime}\right), \psi\left(K^{\prime}\right) \subset$ $L^{\prime}$. If $\varphi$ and $\psi$ are contiguous then their restrictions $\varphi^{\prime}, \psi^{\prime}: K^{\prime} \rightarrow L^{\prime}$ are contiguous too.

The concept of contiguity class provides a simplicial analogue of homotopy classes of continuous maps between topological spaces. By means of the notion of contiguity we define the key concept of categorical subcomplex.

Definition 2.2. Let $K$ be an abstract simplicial complex. We say that the subcomplex $U \subset K$ is categorical (in $K$ ) if there exists a vertex $v \in K$ such that the inclusion $i=i_{U}: U \rightarrow K$ and the constant map $c=c_{v}: U \rightarrow$ $K$ are in the same contiguity class (denoted by $i \sim c$ ).

In other words, the inclusion of $U$ factors through the vertex $v \in K$ up to "simplicial homotopy" (meaning contiguity class). Notice that a categorical subcomplex may not be connected.

Definition 2.3. Let $K$ be an abstract simplicial complex. The simplicial $L S$-category of $K$, denoted by scat $K$, is the least integer $n \geq 0$ such that there exist $n+1$ categorical subcomplexes $U_{0}, \ldots, U_{n}$ of $K$ which cover $K$, that is, such that $K=U_{0} \cup \cdots \cup U_{n}$.

For instance, as we shall explain in Subsection 2.3, scat $K=0$ if and only if $K$ is strongly collapsible to a point, in the sense given by Barmak and Minian $[\mathbf{3}, \mathbf{4}]$.

2.2. Maximal simplices. We now show that the simplicial LS-category can be obtained by taking into account only maximal simplices in its definition. The proof is inspired on the analogous idea for the LS-category of finite topological spaces, that was pointed out to us by J. Strom.

Lemma 2.4. Let $V \subset U \subset K$ be subcomplexes of $K$. If $U$ is categorical (in $K$ ) then $V$ is categorical (in $K$ ).

Proof: If the inclusion $i: U \subset K$ verifies $i \sim c$ for some constant map $c: U \rightarrow K$, and $j: V \subset U$ is the inclusion, then $i \circ j: V \rightarrow K$ is the inclusion, $c \circ j: V \rightarrow K$ is a constant map, and $c \circ j \sim i \circ j$. 
Proposition 2.5. In Definition 2.3 one may assume that:

1. Each categorical subcomplex in the covering $U_{0}, \ldots, U_{n}$ is a union of maximal simplices of $K$.

2. Each maximal simplex of $K$ is contained in only one of the elements of the covering.

Proof: Let $U_{0}, \ldots, U_{n} \subset K$ be a categorical covering. We shall replace each subcomplex $U_{j}$ by another subcomplex $V_{j}$ (may be empty) defined as the union of the simplices $\sigma \in U_{j}$ which are maximal in $K$.

First, $V_{0}, \ldots, V_{n}$ is a covering of $K$, because if $\mu$ is a simplex of $K$, it must be contained in some maximal simplex $\sigma$, which turns out to be contained in some $U_{j}$. Then $\mu \subset \sigma \in V_{j}$, so $\mu \in V_{j}$. Moreover, $V_{j}$ is categorical (by Lemma 2.4), because $V_{j} \subset U_{j}$.

The second part follows from the fact that if we remove each maximal simplex from all excepting one of the $V_{j}$, then the resulting subcomplexes are still categorical, by Lemma 2.4 , and they cover $K$.

2.3. General properties. We state several general properties of simplicial LS-category.

In [4] (see also [3]), Barmak and Minian introduced the notion of strong collapse, a particular type of simplicial collapse in the Whitehead's sense (see Chapter 1, Section 2 of [5]) which is specially adapted to the simplicial structure. Actually, a strong collapse can be modelled as a simplicial map, in contrast with the general concept of simplicial collapse.

Definition 2.6. A vertex $v$ of a simplicial complex $K$ is dominated by another vertex $v^{\prime}$ if every maximal simplex that contains $v$ also contains $v^{\prime}$.

An elementary strong collapse consists of removing the open star of a dominated vertex $v$ from a simplicial complex $K$ (see Figure 1).
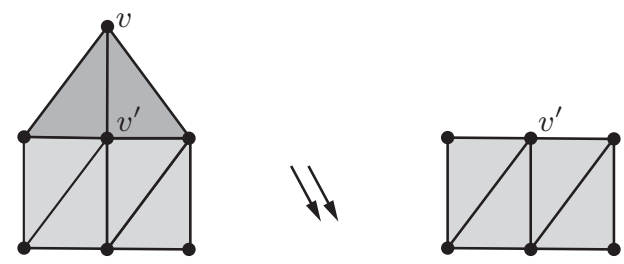

Figure 1. An elementary strong collapse.

The inverse of a strong collapse is called a strong expansion. Then, two simplicial complexes $K, L$ have the same strong homotopy type, 
denoted by $K \sim L$, if they are related by a sequence of strong collapses and expansions. Surprisingly, this turns out to be intimately related to the classical notion of contiguity. More precisely, having the same strong homotopy type is equivalent to the existence of simplicial maps $\varphi: K \rightarrow$ $L$ and $\psi: L \rightarrow K$ such that $\psi \circ \varphi \sim 1_{K}$ and $\varphi \circ \psi \sim 1_{L}$ (see $[4$, Corollary 2.12]). The strong homotopy type gives a simplicial analogue to the homotopy type of topological spaces.

Notice that scat $K=0$ if and only if $K$ is strongly collapsible, that is, there is a finite sequence of elementary strong collapses reducing it to a vertex.

Example 2.7. The simplicial complex in Figure 2 is not strong collapsible, in fact scat $K=1$.

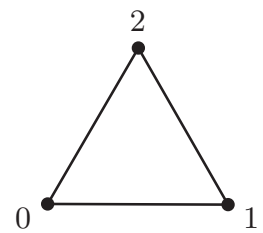

Figure 2. A complex $K$ with scat $K=1$.

For the next example, let us recall the simplicial notions of star and link. Roughly speaking, they correspond to the simplicial counterparts of closed ball and sphere around a vertex. Being more precise, given a simplicial complex $K$ and $v \in K$ a vertex, the star of $v$ in $K$, denoted by $\operatorname{st}(v ; K)$, is the subcomplex of simplices $\sigma \in K$ such that $\sigma \cup\{v\} \in K$. The link of $v$ in $K$, denoted by $\operatorname{lk}(v ; K)$, is the subcomplex of $\operatorname{st}(v ; K)$ of simplices which do not contain $v$.

Example 2.8. Let $K$ be a finite simplicial complex and let $a$ be a vertex not in $K$. The cone $K * a$ is the simplicial complex generated by the simplices of the form $\left(v_{0}, \ldots, v_{n}, a\right)$ where $\left(v_{0}, \ldots, v_{n}\right)$ is a simplex of $K$. Notice that $K * a$ is strong collapsible, that is, $\operatorname{scat}(K * a)=0$. This result can be proved just by taking into account that $\operatorname{lk}(u, K * a)=\operatorname{lk}(u, K) * a$ (see $[\mathbf{2}])$.

Example 2.9. Let $K$ be a finite simplicial complex such that scat $K>$ 0 , and let $S^{0}$ be the complex given by only two 0 -simplices and no other simplices. We define the suspension of $K$ as $\Sigma K=K * S^{0}$. Then $\operatorname{scat}(\Sigma K)=1$. Notice that any suspension can be naturally decomposed as the union of two simplicial cones, and thus scat $(K) \leq 1$. Moreover in this case $\operatorname{scat}(\Sigma) \neq 0$. First, assume that $K$ is a minimal complex, that is 
$K$ has no dominated vertices. The maximal simplices of $\Sigma K$ are $\left(w_{i}, a\right)$ and $\left(w_{i}, a^{\prime}\right)$ where $w_{i}, i=0, \ldots, n$ are the maximal simplices of $K$ and $a, a^{\prime}$ are the 0 -simplices of $S^{0}$. Now, since $K$ has no dominated vertices then for every pair $(v, u)$ of vertices in $K$ there is a maximal simplex $w_{i}$ in $K$ containing $v$ but not containing $u$. Therefore, the maximal simplex $\left(w_{i}, a\right)$ in $\Sigma K$ contains $v$ but not $u$. So $v$ and $u$ are not dominated vertices in the suspension. Moreover any vertex $v$ is not dominated by the vertices $a$ (resp. $a^{\prime}$ ) since there is always a maximal simplex containing $v$ but not $a\left(x\right.$ resp. $\left.a^{\prime}\right)$, meaning the simplex containing $v$ and $a^{\prime}$ (resp. a). Now assume that $K$ is not minimal, then there is a sequence of strong collapses and expansions from $K$ to its core $K^{\prime}$. Since a point that is dominated in $K$ is also dominated in $\Sigma K$, then there is a sequence of strong collapses and expansions from $\Sigma K$ to its core $\Sigma K^{\prime}$. Therefore, by means of the next theorem, $\Sigma K$ and $\Sigma K^{\prime}$ have the same strong homotopy type and the result follows.

The next theorem was proved in $[\mathbf{9}$, Theorem 3.4] by the authors.

Theorem 2.10. The simplicial LS-category is an invariant of the strong homotopy type, that is, $K \sim L$ implies scat $K=$ scat $L$.

Therefore a simplicial complex $K$ and its core $K_{0}$ have the same simplicial LS-category. Remember that the core of a complex is the minimal subcomplex obtained by eliminating dominated vertices (see for instance Barmak's book [3]). More precisely, if a vertex $v$ is dominated by another vertex $v^{\prime}$, then the collapse $r: K \rightarrow K \backslash v$ is a strong equivalence. Under a finite number of steps one attains a complex $K_{0}$ which is minimal, that is, it does not have dominated vertices. This minimal complex is unique up to simplicial isomorphism.

The next result establishes two combinatorial upper bounds for the simplicial category which do not exist for the usual LS-category.

Corollary 2.11. scat $K$ is strictly bounded from above by both:

1. The number of vertices of $K$.

2. The number of maximal simplices of its core $K_{0}$.

Proof: The star of a vertex $\operatorname{st}(v), v \in K_{0}$, is a strong collapsible subcomplex of $K_{0}$ because all vertices in $\operatorname{st}(v)$ are dominated by $v$. Therefore, the family $\left\{\operatorname{st}(v): v \in K_{0}\right\}$ provides a cover of $K_{0}$ by categorical subcomplexes, and so we have that scat $K_{0}<m$, where $m$ is the number of vertices of $K_{0}$. Since the value of scat $K$ is a strong homotopy invariant, scat $K=$ scat $K_{0}<m$.

On the other hand, let $M\left(K_{0}\right)$ be the number of maximal simplices of $K_{0}$. Then, from Proposition 2.5 it follows that scat $K_{0}<M\left(K_{0}\right)$ because any maximal simplex is a strong collapsible subcomplex of $K_{0}$. 
2.4. Geometric simplicial category. In $[\mathbf{9}]$, the authors also introduced the notion of geometric simplicial category gscat $K$ of the complex $K$, which is the analogue of the geometric LS-category of a topological space introduced by Fox in $[\mathbf{1 0}]$, see also $[\mathbf{6}, \S 3.1]$. The difference with scat $K$ is that each subcomplex in a categorical covering is required to be strongly collapsible in itself, rather than in the ambient complex; in other words, the identity, rather than the inclusion, is in the contiguity class of a constant map. Clearly, scat $K \leq$ gscat $K$. However, as in the continuous case, there are complexes where this inequality is strict; for example, in the standard triangulation of the 2-dimensional torus. Geometric simplicial category has a very different behaviour from that of the ordinary category. In particular it is neither hereditary nor homotopically invariant. However, the bounds of Corollary 2.11 are still true for gscat $K$ because gscat $K_{0}=\max \{$ gscat $L: L \sim K\}$, as proven in $[\mathbf{9}]$.

Definition 2.12. Let $K$ and $L$ be two finite simplicial complexes. We define the join $K * L$ as the simplicial complex with set of vertices $K^{0} \cup$ $L^{0}$ and with simplices the simplices of $K$, the simplices of $L$, and the simplices given by $\sigma \cup \tau, \sigma$ simplex in $K$ and $\tau$ simplex in $L$.

Proposition 2.13. Let $K, L$ be two finite simplicial complexes, then $\operatorname{gscat}(K * L) \leq \min \{$ gscat $K$, gscat $L\}$.

Proof: Suppose that $\min \{$ gscat $K$, gscat $L\}=$ gscat $K=n$, then there are $n+1$ strong collapsible subcomplexes $U_{0}, \ldots, U_{n}$ covering $K$. Consider the subcomplexes $U_{0} * L, \ldots, U_{n} * L$. These subcomplexes are strong collapsible [3, Proposition 5.1.16] and they provide a cover of $K * L$.

\section{Barycentric subdivision}

We now study the behaviour of scat under barycentric subdivisions. Our main result states that scat is decreasing under such kind of subdivisions.

Theorem 3.1. Let $\mathrm{sd} K$ be the first barycentric subdivision of the simplicial complex $K$. Then $\operatorname{scat}(\operatorname{sd} K) \leq \operatorname{scat} K$.

This theorem was proved in [9, Corollary 6.7] using results of Barmak and Minian (precisely, [4, Proposition 4.11 and Proposition 4.12]) about finite spaces. We shall reformulate them in order to give a direct proof.

If $K$ is an abstract simplicial complex, its first barycentric subdivision can be defined formally as the complex $\operatorname{sd} K$ whose vertices $\{\sigma\}$ are identified to the simplices $\sigma=\left\{v_{0}, \ldots, v_{p}\right\}$ of $K$, while the simplices of $\operatorname{sd} K$ are the sequences $\left\{\sigma_{1}, \ldots, \sigma_{q}\right\}$ of simplices of $K$ such that $\sigma_{1} \subset$ $\cdots \subset \sigma_{q}($ see $[\mathbf{1 6}, \S 2.1])$. 
Definition 3.2. Let $\varphi: K \rightarrow L$ be a simplicial map. The induced map $\operatorname{sd} \varphi: \operatorname{sd} K \rightarrow \operatorname{sd} L$ is defined as

$$
(\operatorname{sd} \varphi)\left(\left\{\sigma_{1}, \ldots, \sigma_{q}\right\}\right)=\left\{\varphi\left(\sigma_{1}\right), \ldots, \varphi\left(\sigma_{q}\right)\right\} .
$$

Clearly $\operatorname{sd} \varphi$ is a simplicial map, $\operatorname{sdid}=\mathrm{id}$ and $\operatorname{sd}(\varphi \circ \psi)=(\operatorname{sd} \varphi) \circ$ $(\operatorname{sd} \psi)$.

Proposition 3.3. If the simplicial maps $\varphi, \psi: K \rightarrow L$ are in the same contiguity class, $\varphi \sim \psi$, then $\operatorname{sd} \varphi, \operatorname{sd} \psi: \operatorname{sd} K \rightarrow \operatorname{sd} L$ are in the same contiguity class, $\operatorname{sd} \varphi \sim \operatorname{sd} \psi$.

Proof: Without loss of generality, we may assume that the maps $\varphi$ and $\psi$ are contiguous, $\varphi \sim_{c} \psi$, which means that $\varphi(\sigma) \cup \psi(\sigma)$ is a simplex, for any simplex $\sigma \in K$. Let $F: \operatorname{sd} K \rightarrow \operatorname{sd} L$ be the map given by

$$
F\left(\left\{\sigma_{1}, \ldots, \sigma_{q}\right\}\right)=\left\{\varphi\left(\sigma_{1}\right) \cup \psi\left(\sigma_{1}\right), \ldots, \varphi\left(\sigma_{q}\right) \cup \psi\left(\sigma_{q}\right)\right\} .
$$

We shall prove that $\operatorname{sd} \varphi \sim F$ by increasing step by step the size of the set

$$
\Omega(\operatorname{sd} \varphi, F)=\{\sigma \in K:(\operatorname{sd} \varphi)(\{\sigma\})=F(\{\sigma\})\} .
$$

Note that $(\operatorname{sd} \varphi)(\{\sigma\})=\{\varphi(\sigma)\}$, while $F(\{\sigma\})=\{\varphi(\sigma) \cup \psi(\sigma)\}$.

If $\operatorname{sd} \varphi=F$ there is nothing to prove. Otherwise, there exists $\mu \in K$ such that $\mu \notin \Omega(\operatorname{sd} \varphi, F)$, or equivalently, $\varphi(\mu)$ is strictly contained in $\varphi(\mu) \cup \psi(\mu)$. Let us take $\mu$ to be of maximal dimension with this property; in this way $\varphi(\sigma)=\varphi(\sigma) \cup \psi(\sigma)$ when $\mu$ is a proper face of $\sigma$. Now, we can define a new map $F_{1}: \operatorname{sd} K \rightarrow \operatorname{sd} L$ as

$$
F_{1}(\{\sigma\})= \begin{cases}(\operatorname{sd} \varphi)(\{\sigma\}) & \text { if } \sigma \neq \mu \\ F(\{\mu\}) & \text { if } \sigma=\mu\end{cases}
$$

It follows:

(1) The map $F_{1}$ is simplicial. In fact, if $\left\{\sigma_{1}, \ldots, \sigma_{q}\right\}$ is a simplex of sd $K$, then

$$
F_{1}\left(\left\{\sigma_{1}, \ldots, \sigma_{q}\right\}\right)=\left\{\varphi\left(\sigma_{1}\right), \ldots, \varphi\left(\sigma_{q}\right)\right\}
$$

if $\sigma_{j} \neq \mu$ for all $j=1, \ldots, q$, while

$$
F_{1}\left(\left\{\sigma_{1}, \ldots, \sigma_{q}\right\}\right)=\left\{\varphi\left(\sigma_{1}\right), \ldots, \varphi(\mu) \cup \psi(\mu\}, \ldots, \varphi\left(\sigma_{q}\right)\right\},
$$

if $\sigma_{j}=\mu$ for some $j$. In both cases the image is a simplex of $\operatorname{sd} L$. Note that in the second case, by the maximality of $\mu$ cited above, it follows that $\varphi(\mu) \cup \psi(\mu) \subset \varphi\left(\sigma_{i}\right)$ if $i>j$. 
(2) We have $\operatorname{sd} \varphi \sim_{c} F_{1}$, because if $\left\{\sigma_{1}, \ldots, \sigma_{q}\right\} \in \operatorname{sd} K$ then

$$
\begin{aligned}
(\operatorname{sd} \varphi)\left(\left\{\sigma_{1}, \ldots, \sigma_{q}\right\}\right) & \cup F_{1}\left(\left\{\sigma_{1}, \ldots, \sigma_{q}\right\}\right) \\
& =\left\{\varphi\left(\sigma_{1}\right), \ldots, \varphi\left(\sigma_{q}\right)\right\} \cup\left\{F_{1}\left(\left\{\sigma_{1}\right\}\right), \ldots, F_{1}\left(\left\{\sigma_{q}\right\}\right)\right\},
\end{aligned}
$$

which equals the simplex

$$
\left\{\varphi\left(\sigma_{1}\right), \ldots, \varphi\left(\sigma_{j-1}\right), \varphi\left(\sigma_{j}\right) \cup \psi\left(\sigma_{j}\right), \ldots, \varphi\left(\sigma_{q}\right) \cup \psi\left(\sigma_{q}\right)\right\},
$$

where $j$ is the lowest index such that $\sigma_{j}=\mu$, if such a $j$ exists.

(3) Finally, $\Omega(\operatorname{sd} \varphi, F) \varsubsetneqq \Omega\left(F_{1}, F\right)$, by the definition of $F_{1}$.

By repeating this construction we shall obtain a sequence of contiguous maps $\operatorname{sd} \varphi \sim_{c} F_{1} \sim_{c} \cdots \sim_{c} F$, which shows that $\operatorname{sd} \varphi \sim F$. Using the same argument for $\psi$, we can prove that $\operatorname{sd} \psi \sim F$. Thus $\operatorname{sd} \varphi \sim \operatorname{sd} \psi$, as claimed.

Remark 3.4. However, subdivision does not preserve the strong homotopy type, as shown by the following example taken from [3, Example 5.1.13]. Consider $K$ to be the boundary of a 2 -simplex and $\operatorname{sd} K$ its barycentric subdivision as in Figure 3. They are both minimal complexes because they have no dominated vertices, but they are not isomorphic, therefore they do not have the same strong homotopy type (see Lemma 6.9).
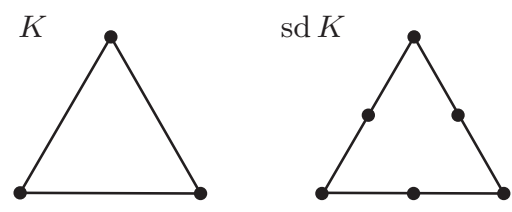

Figure 3. The simplicial complex $K$ does not have the same strong homotopy type of its subdivision sd $K$.

Proof of Theorem 3.1: Let scat $K=n$, and take a categorical covering $U_{0}, \ldots, U_{n}$ of $K$. Consider the subcomplexes sd $U_{0}, \ldots, \operatorname{sd} U_{n}$, which cover $\operatorname{sd} K$. Since each inclusion $I_{j}: U_{j} \subset K$ is in the contiguity class of some constant map $v_{j}: U_{j} \rightarrow K$, denoted by $I_{j} \sim v_{j}$, it follows from Proposition 3.3 that $\operatorname{sd} I_{j} \sim \operatorname{sd} v_{j}$. But it is clear that $\operatorname{sd} I_{j}$ is the inclusion $\operatorname{sd} U_{j} \subset \operatorname{sd} K$, while sd $v_{j}$ is the constant map $\left\{v_{j}\right\}$. Then each $\operatorname{sd} U_{j}$ is a categorical subcomplex and $\operatorname{scat}(\operatorname{sd} K) \leq n$.

Corollary 3.5. Let $K$ be a finite simplicial complex and let $\mathrm{sd} K$ be the first barycentric subdivision. Then, scat $K=1$ implies that $\operatorname{scat}(\operatorname{sd} K)=$ 1. 
Proof: In [4, Theorem 4.15] it is proved that a complex $K$ is strongly collapsible if and only if sd $K$ is strongly collapsible. In other words, scat $K=0$ if and only if scat $(\operatorname{sd} K)=0$. Jointly with our Theorem 3.1 this ends the proof.

Example 3.6. The following example, suggested to the third author by J. Barmak, shows a complex where the inequality of Theorem 3.1 is strict.

Let $K$ be the complete graph $K_{5}$ (see Figure 4$)$ considered as a 1-dimensional simplicial complex.

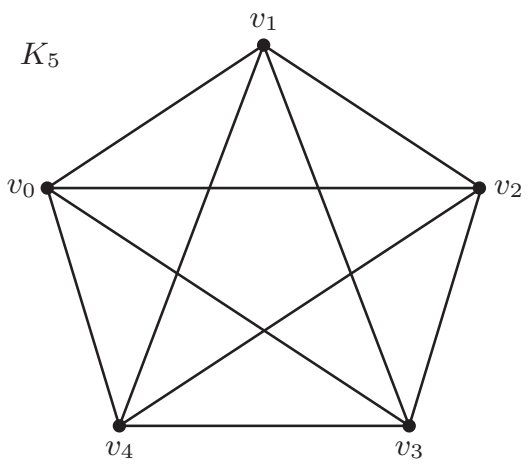

Figure 4. The graph $K=K_{5}$ verifies scat $(\operatorname{sd} K)<\operatorname{scat} K$.

Let us consider the following categorical cover of $K$ :

$$
\begin{aligned}
& U_{0}=v_{0} v_{1} \cup v_{0} v_{2} \cup v_{0} v_{3} \cup v_{0} v_{4}, \\
& U_{1}=v_{1} v_{4} \cup v_{1} v_{2} \cup v_{2} v_{3}, \\
& U_{2}=v_{1} v_{3} \cup v_{3} v_{4} \cup v_{2} v_{4} .
\end{aligned}
$$

Therefore scat $K_{5} \leq 2$. Moreover, there is no cover of two categorical subcomplexes. In fact, if it happens, then one of the two subcomplexes has to contain at least 5 edges. Moreover, any tree contains one more vertex than the number of edges. Hence, any forest with 5 edges should have at least 6 vertices, which is impossible in our complex.

We conclude that there is not any categorical subcomplex with at least 5 vertices, therefore there is no covering of $K_{5}$ given by two categorical subcomplexes. Hence we have scat $K=2$.

On the other hand, the first barycentric subdivision sd $K$ has a covering with two categorical subcomplexes given, for example, by the two 
subcomplexes that are showed in Figure 5: $L_{0}$, drawn with continuous edges, and $L_{1}$, drawn with dashed edges. Since sd $K_{5}$ is not strongly collapsible we can conclude that $1=\operatorname{scat}\left(\operatorname{sd} K_{5}\right)<\operatorname{scat} K_{5}=2$.

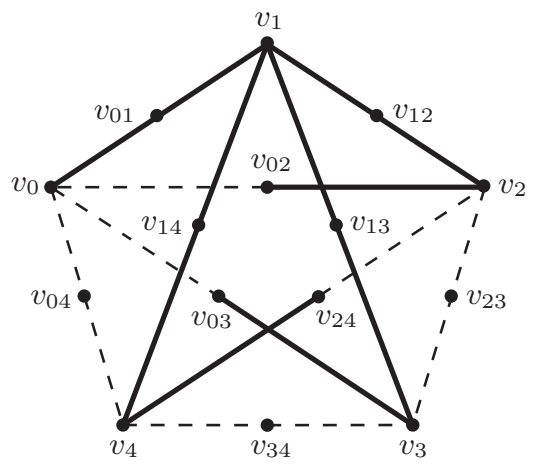

FiguRE 5. A covering of sd $K_{5}$ with two categorical subcomplexes.

Remark 3.7. Note that scat $K_{5}$ equals the arboricity of $K_{5}$ minus one; later we shall prove that this is a general result for any graph (Theorem 8.12).

It is interesting to point out that Theorem 3.1 can be extended to the geometrical simplicial category, that is, gscat(sd $K) \leq \operatorname{gscat} K$ with an analogous argument. In addition, notice that the complex sd $K_{5}$ (Figure 5) can be covered with two trees and thus, gscat $\left(\operatorname{sd} K_{5}\right)=1$.

\section{Geometric realization}

A natural question is to compare the simplicial category of the complex $K$ with the LS-category of $X=|K|$, the so-called geometric realization of $K[\mathbf{2 0}, \S 3.1]$.

We recall the classical definition of Lusternik-Schnirelmann category of a topological space. For general properties of this invariant we refer to $[6]$.

Definition 4.1. An open subset $U$ of the topological space $X$ is called categorical if $U$ can be contracted to a point inside the ambient space $X$. The $L S$-category of $X$, denoted by cat $X$, is the least integer $n \geq 0$ such that there is a covering of $X$ by $n+1$ categorical open subsets. 
It is known (see Proposition 1.10 of [6]) that, when the space $X$ is a normal ANR, the categorical sets in Definition 4.1 can be taken to be closed instead of open. In particular, this is the case for the geometric realization $X=|K|$ of a finite abstract simplicial complex (see $[\mathbf{1 7}$, $\S$ II.4], also [7, p. 84] and the references and comments in [15, p. 247]).

On the other hand, O. Randal-Wallis pointed out to the second author the following example, showing that categorical sets can be rather pathological.

Example 4.2. Let $X=[0,1]$ be the unit interval and let $F$ be the Cantor set. Then $F$ is contractible in $X$ and has not the homotopy type of a finite CW-complex. This is because $F$ is totally disconnected but non-discrete (see $[\mathbf{1 1}, \S 5.1]$ ).

Anyway, the following theorem shows that, when $X=|K|$ is the geometric realization of an abstract simplicial complex $K$, the LS-category of $X$ can be computed by means of a closed categorical covering whose sets are subcomplexes of $K$ in a certain subdivision. This result is essentially stated, but without proof, in Fox's paper [10, §3].

Theorem 4.3. If $X=|K|$ then cat $X \leq n$ if and only if there exist subcomplexes $L_{0}, \ldots, L_{n}$ of some subdivision $K^{\prime}$ of $K$, such that each $\left|L_{j}\right|$ is contractible in $X$ and $X=\left|L_{0}\right| \cup \cdots \cup\left|L_{n}\right|$.

The proof of the above Theorem 4.3 can be sketched as follows: a categorical covering of $X$ has a Lebesgue number $\delta$. Recall that the mesh of a simplicial complex is the supremum of the diameters of all its simplices [20]. If we take a subdivision $\operatorname{sd}^{n} K$ of $K$ such that $\operatorname{mesh}\left(\operatorname{sd}^{n} K\right)<\delta$, then, by considering the subcomplexes formed by the simplices contained on each element of this covering, a categorical covering by subcomplexes is obtained.

We now state the precise relation between the simplicial category of a complex and the topological LS-category of its geometric realization.

Theorem 4.4. Let $K$ be a finite simplicial complex. Then cat $|K| \leq$ scat $K$.

Proof: Let scat $K=n$ and let $\left\{U_{0}, \ldots, U_{n}\right\}$ be a categorical simplicial covering of $K$, that is, each inclusion $I_{j}: U_{j} \rightarrow K$ is in the same contiguity class that a constant map $v_{j}$. Therefore, the induced maps $\left|I_{j}\right|,\left|v_{j}\right|:\left|U_{j}\right| \rightarrow|K|$ between the geometric realizations are homotopic, $\left|I_{j}\right| \simeq\left|v_{j}\right|($ see $[\mathbf{2 0}, \S 3.4])$. It is clear that $\left|I_{j}\right|$ is the inclusion $\left|U_{j}\right| \subset|K|$, and $\left|v_{j}\right|$ is a constant map. Thefore the subspaces $\left|U_{0}\right|, \ldots,\left|U_{n}\right|$ form a categorical closed cover of $|K|$. As we commented before, since $|K|$ is a 
normal ANR we can consider closed covers instead of open covers. Then cat $|K| \leq n$.

Corollary 4.5. cat $|K| \leq \operatorname{scat}\left(\mathrm{sd}^{N} K\right)$, for any iterated barycentric subdivision $\mathrm{sd}^{N} K$ of the complex $K$.

Proof: The geometric realizations $|\mathrm{sd} K|$ and $|K|$ are homeomorphic (see for instance [16, Proposition 2.33]).

Example 4.6. The inequality in Corollary 4.5 may be strict. For instance, the complex $K$ in Figure 6 is topologically contractible (it is collapsible), that is cat $|K|=0$, but all the barycentric subdivisions have simplicial category one.

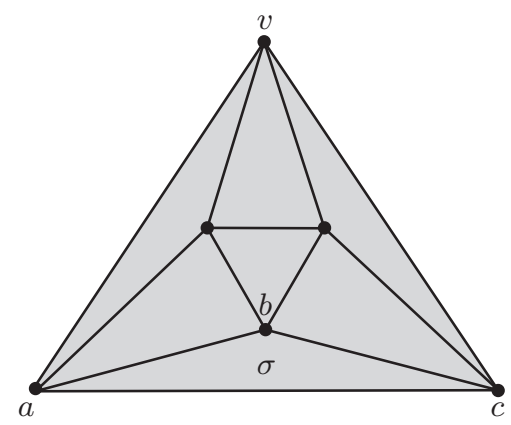

Figure 6. A collapsible but not strongly collapsible complex $K$. The subcomplex $L=K \backslash \sigma$ does not have the contiguity extension property.

This happens because, as proven by Barmak and Minian in [4, Theorem 4.15], a complex is strongly collapsible if and only if its barycentric subdivision is strongly collapsible. In other words, scat $K=0$ if and only if $\operatorname{scat}(\operatorname{sd} K)=0$. But the complex $K$ is not strongly collapsible (it has no dominated vertices) and can be covered by two categorical subcomplexes, so scat $K=1$. By applying Theorem 3.1 we have $0<\operatorname{scat}\left(\operatorname{sd}^{N} K\right) \leq 1$ for all $N$.

\section{Products}

We recall the definition of categorical product (see Remark 5.2 below for an explanation of our notations). 
Definition 5.1. Let $K, L$ be two abstract simplicial complexes. The categorical product $K \times L$ is defined as follows. The vertices of $K \times L$ are the pairs $(v, w)$ of vertices with $v \in K$ and $w \in K$. The simplices of $K \times L$ are the sets of vertices $\left\{\left(v_{1}, w_{1}\right), \ldots,\left(v_{q}, w_{q}\right)\right\}$ such that $\left\{v_{1}, \ldots, v_{q}\right\}$ is a simplex of $K$ and $\left\{w_{1}, \ldots, w_{q}\right\}$ is a simplex of $L$.

We fix some notation.

For $n \geq 1$, we shall denote $K^{n}=K \times \stackrel{n)}{.} \times K$. By definition, the projections $\pi_{j}: K \rightarrow K^{n}, j=1, \ldots, n$, onto each factor are simplicial maps. The diagonal map $\Delta: K \rightarrow K^{n}$ defined by $\Delta(v)=(v, \ldots, v)$ is simplicial too. A map $L \rightarrow K^{n}$ is simplicial if and only if the compositions with all the projections are simplicial maps.

If $\varphi_{1}, \ldots, \varphi_{n}: K \rightarrow L$ are simplicial maps, the map $\left(\varphi_{1}, \ldots, \varphi_{n}\right): K \rightarrow$ $L^{n}$ defined by

$$
\left(\varphi_{1}, \ldots, \varphi_{n}\right)(v)=\left(\varphi_{1}(v), \ldots, \varphi_{n}(v)\right)
$$

is simplicial.

If $\varphi: K \rightarrow L$ is a simplicial map then we denote by $\varphi^{n}: K^{n} \rightarrow L^{n}$ the map

$$
\varphi^{n}\left(v_{1}, \ldots, v_{n}\right)=\left(\varphi\left(v_{1}\right), \ldots, \varphi\left(v_{n}\right)\right),
$$

which is simplicial.

Remark 5.2. Our Definition 5.1 of categorical product is Definition 4.25 in [16], but we changed the notation $K \prod L$ to $K \times L$ for the sake of simplicity. This categorical (or simplicial) product should not be confused with the so-called cartesian product [8, §II.8], which depends on some ordering of the vertices and does not verify the universal property of a product. As an example, the categorical product $\Delta^{1} \times \Delta^{1}$ is isomorphic to $\Delta^{3}$, while the cartesian product is $\Delta^{2}$. Also note that $\left|K^{n}\right|$ is not homeomorphic to $|K|^{n}$. However, as Kozlov proves in [16, Proposition 15.23], the geometric realizations of both products have the same homotopy type, so $\left|K^{n}\right| \simeq|K|^{n}$ (see also [16, Theorem 10.21]).

The next proposition establishes two natural results about contiguity of maps on categorical products that can be proved in a straightforward way.

Proposition 5.3. 1. Let $\varphi, \psi: K \rightarrow L$ and $\varphi^{\prime}, \psi^{\prime}: K^{\prime} \rightarrow L^{\prime}$ be simplicial maps such that $\varphi \sim_{c} \psi$ and $\varphi^{\prime} \sim_{c} \psi^{\prime}$. Then $\varphi \times \varphi^{\prime} \sim_{c}$ $\psi \times \psi^{\prime}: K \times K^{\prime} \rightarrow L \times L^{\prime}$.

2. Let $\varphi \sim_{c} \psi: K \rightarrow L$ and $\varphi^{\prime} \sim_{c} \psi^{\prime}: K \rightarrow L^{\prime}$, then $\left(\varphi, \varphi^{\prime}\right) \sim_{c}$ $\left(\psi, \psi^{\prime}\right): K \rightarrow L \times L^{\prime}$. 
Corollary 5.4. Let $K \sim L$ be two complexes with the same strong homotopy type. Then $K^{n} \sim L^{n}$.

At this point we are in conditions to prove the main result of this section. It is interesting to point out that the corresponding result for the classical LS-category is cat $(X \times Y) \leq$ cat $X+$ cat $Y$ when the topological space $X \times Y$ is completely normal [6, Theorem 1.37], while the result cat $E+1 \leq($ cat $B+1)($ cat $F+1)$ is true for an arbitrary fibration $[\mathbf{6}$, Theorem 1.41].

Theorem 5.5. Let $K$ and $L$ be finite simplicial complexes. Then

$$
\operatorname{scat}(K \times L)+1 \leq(\operatorname{scat} K+1)(\operatorname{scat} L+1) .
$$

Proof: Suppose that scat $K=n$ and scat $L=m$, therefore there exists a categorical covering $U_{0}, \ldots, U_{n}$ of $K$ and a categorical covering $V_{0}, \ldots, V_{m}$ of $L$. Consider the subcomplexes $U_{i} \times V_{j} \subset K \times L$, for $0 \leq i \leq n$ and $0 \leq j \leq m$. We want to show that $U_{i} \times V_{j}$ form a categorical covering of $K \times L$.

Each inclusion map $i_{U_{i}}$ is in the same contiguity class of a constant map $c_{u_{i}}$ where $u_{i}$ is a vertex of $K$; analogously, each inclusion $i_{V_{j}}$ is in the same contiguity class of a constant map $c_{v_{j}}$ where $v_{j}$ is a vertex in $L$. By Proposition 5.3 the map $i_{U_{i}} \times i_{V_{j}}: U_{i} \times V_{j} \rightarrow K \times L$ is in the same contiguity class of the map $c_{v_{i}} \times c_{w_{j}}: U_{i} \times V_{j} \rightarrow K \times L$. Clearly, $i_{U_{i}} \times i_{W_{j}}=i_{U_{i} \times V_{j}}$ and $c_{v_{i}} \times c_{w_{j}}=c_{\left(v_{i}, w_{j}\right)}$, where $\left(v_{i}, w_{j}\right)$ is a vertex of $K \times L$. Therefore the subcomplexes $U_{i} \times V_{j}$ are categorical.

Now, we shall prove that $\left\{U_{i} \times V_{j}\right\}$ is a covering of $K \times L$. If $\left\{\left(v_{0}, w_{0}\right), \ldots,\left(v_{q}, w_{q}\right)\right\}$ is a simplex of $K \times L$ then $\left\{v_{0}, \ldots, v_{q}\right\}$ is contained in a subcomplex $U_{i}$ of $K$ and $\left\{w_{0}, \ldots, w_{q}\right\}$ is contained in a subcomplex $W_{j}$ of $L$. Then $\left\{\left(v_{0}, w_{0}\right), \ldots,\left(v_{q}, w_{q}\right)\right\}$ is contained in $U_{i} \times V_{j}$. Thus, we conclude that $\operatorname{scat}(K \times L)+1 \leq(n+1)(m+1)$.

\section{Whitehead construction}

It is well known that for topological spaces $X$ with "good properties" there is the following so-called Whitehead characterization of the topological LS-category (see [6, Theorem 1.55]):

Theorem 6.1. cat $X \leq n$ if and only if the diagonal map $\Delta: X \rightarrow X^{n+1}$ factors (up to homotopy) through the so-called fat wedge $T^{n+1} X$.

This result is a very useful tool for computing LS-category. In this section we shall try to adapt it to abstract simplicial complexes. 
First we define a simplicial version of the topological fat wedge $T^{n} X$ of a topological space $X[\mathbf{6}, \S 1.6]$. Also, we shall briefly develop the notion of pointed contiguity class.

Let $K$ be an abstract simplicial complex and fix some vertex $v_{0}$ of $K$ as a base point. For each $j=1, \ldots, n$ let

$$
K_{j}=\pi_{j}^{-1}\left(\left\{v_{0}\right\}\right)=K \times \cdots \times\left\{v_{0}\right\} \times \cdots \times K
$$

be the subcomplex of $K^{n}$ spanned by the vertices whose $j$-th coordinate is the base point $v_{0}$.

Definition 6.2. For $n \geq 1$ the $n$-th fat wedge $T^{n} K$ is the subcomplex $K_{1} \cup \cdots \cup K_{n} \subset K^{n}$.

For instance, $T^{1} K=\left\{v_{0}\right\}$ is a point and $T^{2} K$ is the wedge $K \vee K$. Note that $T^{n} K$ is not a full subcomplex of $K^{n}$.

A pointed map (that is, a simplicial map preserving the base points) $\varphi:\left(K, v_{0}\right) \rightarrow\left(L, w_{0}\right)$ induces a simplicial map $T^{n} \varphi: T^{n} K \rightarrow T^{n} L$, which is the restriction of $\varphi^{n}: K^{n} \rightarrow K^{n}$.

Proposition 6.3. Let $\varphi, \psi: K \rightarrow L$ be two contiguous simplicial maps preserving the base points. Then the induced maps $T^{n} \varphi, T^{n} \psi: T^{n} K \rightarrow$ $T^{n} L$ are contiguous.

Proof: The maps $\varphi^{n}, \psi^{n}: K^{n} \rightarrow L^{n}$ are contiguous by Proposition 5.3. Moreover they send each subcomplex $K_{j}$ into itself.

Corollary 6.4. Let $\left(K, v_{0}\right) \sim\left(L, w_{0}\right)$ be two pointed simplicial complexes with the same pointed strong homotopy type, that is, we assume that the homotopy equivalences $\varphi, \psi$ between $K$ and $L$, as well as the sequences of contiguous maps defining the relations $\psi \circ \varphi \sim 1_{K}$ and $\varphi \circ \psi \sim$ $1_{L}$, preserve the base points. Then $T^{n} K \sim T^{n} L$.

We are now in position to discuss the Whitehead formulation of the simplicial LS-category. In order to be systematic we follow the approach of $[6, \S 1.6]$, by defining a so-called simplicial Whitehead category scat ${ }^{\mathrm{Wh}} K$ and trying to compare it to the simplicial LS-category scat $K$.

Definition 6.5. We say that scat ${ }^{\mathrm{Wh}} K \leq n$ if the diagonal map $\Delta: K \rightarrow$ $K^{n+1}$ factors through the fat wedge $T^{n+1} K$ up to contiguity class. That 
is, there exists some simplicial map $\delta: K \rightarrow T^{n+1} K$ such that $I \circ \delta \sim \Delta$, where we denote by $I: T^{n+1} K \subset K^{n+1}$ the inclusion:

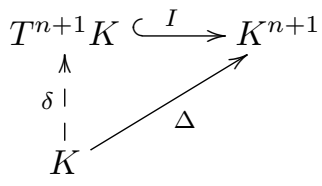

Theorem 6.6. scat $K \leq$ scat $^{\mathrm{Wh}} K$.

Proof: Assume that scat ${ }^{\mathrm{Wh}} K=n$, and let $\delta: K \rightarrow T^{n+1} K$ be as in Definition 6.5 . Let $\pi_{j}: K^{n+1} \rightarrow K$ be the projections onto each factor. Let $K_{j} \subset T^{n+1} K, j=1, \ldots, n+1$, be the subcomplexes defined in (1).

Since $I \circ \delta \sim \Delta$, there exists a sequence $I \circ \delta=\varphi_{1}, \varphi_{2}, \ldots, \varphi_{m}=\Delta$ of maps such that $\varphi_{i}$ and $\varphi_{i+1}$ are contiguous. Call $L_{j}=\Delta^{-1}\left(K_{j}\right)$ the preimages of the $K_{j} \subset T^{n+1} K$ defined above. Clearly, $K=L_{1} \cup$ $\cdots \cup L_{n+1}$. It only remains to show that each subcomplex $L_{j} \subset K$ is categorical, that is, each inclusion map $I_{j}: L_{j} \subset K$ is in the same contiguity class that a constant map.

Since $\varphi_{i} \sim_{c} \varphi_{i+1}: K \rightarrow K^{n+1}$ it follows that

$$
\pi_{j} \circ \varphi_{i} \circ I_{j} \sim_{c} \pi_{j} \circ \varphi_{i+1} \circ I_{j}: L_{j} \rightarrow K
$$

Now, $\pi_{j} \circ \varphi_{1} \circ I_{j}=\pi_{j} \circ I \circ \delta \circ I_{j}$, which is the constant map $c_{v_{0}}$ because $\Delta\left(L_{j}\right) \subset K_{j}$. On the other hand, $\pi_{j} \circ \varphi_{m} \circ I_{j}=\pi_{j} \circ \Delta \circ I_{j}$ is the inclusion $I_{j}$. Then $I_{j} \sim c_{v_{0}}$.

We have found $n+1$ categorical subcomplexes covering $K$, therefore scat $K \leq n$.

In this section we shall prove that the converse inequality of Theorem 6.6 is not true, by exhibiting an example of a complex $K$ such that scat $K=1$ while scat ${ }^{\mathrm{Wh}} K>2$. The proof of the next propositions is inspired by a result about finite co-H-spaces proved in [14].

First we prove that the simplicial Whitehead category is an invariant of the pointed strong homotopy type.

Proposition 6.7. If $\left(K, v_{0}\right) \sim\left(L, v_{0}\right)$ is a pointed strong equivalence as in Corollary 6.4, then scat ${ }^{\mathrm{Wh}} K=$ scat $^{\mathrm{Wh}} L$.

Proof: Let scat ${ }^{\mathrm{Wh}} L=n$. Consider the following diagram and the strong equivalences $K^{n+1} \sim L^{n+1}$ (Corollary 5.4) and $T^{n+1} K \sim T^{n+1} L$ (Corollary 6.4). 


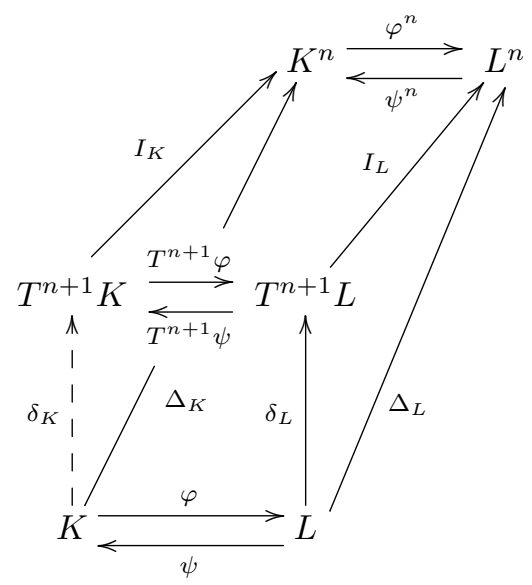

With the obvious notations, from the relations $\psi \circ \varphi \sim 1_{K}$ and $\varphi \circ \psi \sim 1_{L}$ it follows that

$$
\psi^{n+1} \circ \varphi^{n+1} \sim 1_{K^{n+1}}, \quad \varphi^{n+1} \circ \psi^{n+1} \sim 1_{L^{n+1}},
$$

as well as

$$
T^{n+1} \psi \circ T^{n+1} \varphi \sim 1_{T^{n+1} K}, \quad T^{n+1} \varphi \circ T^{n+1} \psi \sim 1_{T^{n+1} L} .
$$

Moreover, from the definitions, we have

$$
I_{L} \circ T^{n+1} \varphi=\varphi^{n} \circ I_{K}, \quad I_{K} \circ T^{n+1} \psi=\psi^{n} \circ I_{L},
$$

as well as

$$
\Delta_{L} \circ \varphi=\varphi^{n} \circ \Delta_{K}, \quad \Delta_{K} \circ \psi=\psi^{n} \circ \Delta_{L}
$$

Define

$$
\delta_{K}:=T^{n+1} \psi \circ \delta_{L} \circ \varphi .
$$

Since, from hypothesis, $I_{L} \circ \delta_{L} \sim \Delta_{L}$, it follows

$$
\begin{aligned}
I_{K} \circ \delta_{K} & =\left(I_{K} \circ T^{n+1} \psi\right) \circ \delta_{L} \circ \varphi \\
& =\psi^{n} \circ\left(I_{L} \circ \delta_{L}\right) \circ \varphi \sim\left(\psi^{n} \circ \Delta_{L}\right) \circ \varphi \\
& =\Delta_{K} \circ(\psi \circ \varphi) \sim 1_{K} .
\end{aligned}
$$

Then scat ${ }^{\mathrm{Wh}} K \leq$ scat $L$. The other inequality is proved in the same way.

Clearly, we may assume that the base point $v_{0}$ is in the core $K_{0}$, hence eliminating dominated vertices is a pointed equivalence $\left(K, v_{0}\right) \sim$ $\left(K_{0}, v_{0}\right)$. 
Corollary 6.8. The simplicial Whitehead category of a complex equals that of its core, scat ${ }^{\mathrm{Wh}} K=$ scat $^{\mathrm{Wh}} K_{0}$.

We are now in a position to prove the main results of this section.

Lemma 6.9 ([4, Proposition 2.7]). Let $K_{0}$ be a minimal complex and let $\varphi: K_{0} \rightarrow K_{0}$ be a simplicial map which lies in the same contiguity class as the identity. Then $\varphi$ is the identity.

Theorem 6.10. Let $K$ be a simplicial complex such that scat ${ }^{\mathrm{Wh}} K \leq 1$. Then $K$ is strongly collapsible, which is equivalent to scat $K=0$.

Proof: If scat ${ }^{\mathrm{Wh}} K=0$ then the result follows from Theorem 6.6.

If scat ${ }^{\mathrm{Wh}} K=1$ we have, from Corollary 6.8 , that scat ${ }^{\mathrm{Wh}} K_{0}=1$. This means that there exists a simplicial map $\varphi: K_{0} \rightarrow T^{2} K_{0}$ such that $i \circ \varphi \sim$ $\Delta$, where $\Delta: K_{0} \rightarrow\left(K_{0}\right)^{2}$ is the diagonal map. Let $\pi_{1}, \pi_{2}:\left(K_{0}\right)^{2} \rightarrow K_{0}$ be the projections of the categorical product. Then

$$
i \circ \varphi \sim \Delta \Longrightarrow \pi_{1} \circ i \circ \varphi \sim \pi_{1} \circ \Delta=1 \Longrightarrow \pi_{1} \circ i \circ \varphi=1
$$

because $K_{0}$ is a minimal complex (Lemma 6.9). Remember that in the proof of Theorem 6.6 we denoted by $L_{1}$ (respectively, $L_{2}$ ) the subcomplex $\varphi^{-1}\left(\left\{v_{0}\right\} \times K\right)\left(\right.$ resp. $\left.\varphi^{-1}\left(K \times\left\{v_{0}\right\}\right)\right)$. Then, for $v \in L_{1}$ we have $v_{0}=\pi_{1} \circ i \circ \varphi(v)=v$ because $\varphi(v) \in L_{1}$, which shows that $L_{1}=\left\{v_{0}\right\}$. Analogously, $\pi_{2} \circ i \circ \varphi=1=\pi_{2} \circ \Delta$ proves that $L_{2}=\left\{v_{0}\right\}$. Then the core $K_{0}=L_{1} \cup L_{2}=\left\{v_{0}\right\}$ is a point, that is, the complex $K$ has the strong homotopy type of a point.

Example 6.11. The complex $K$ in Figure 6 has scat $K=1$ so it is not strongly equivalent to a point. From Theorem 6.10 its Whitehead simplicial category is at least 2 . Then scat ${ }^{\mathrm{Wh}} K>\operatorname{scat} K$.

\section{Cofibrations}

We now briefly discuss the notion of cofibration in the simplicial setting.

The "homotopy extension property" is a very important notion in topology. A cofibration is a map $A \rightarrow X$ which satisfies the homotopy extension property with respect to all spaces. It is well known (see [13, p. 14] or $[\mathbf{1 7}$, p. 68]) that if $A$ is a subcomplex of a CW-complex $X$ then the pair $(X, A)$ has the homotopy extension property. Therefore, if $L \subset K$ is a subcomplex of a simplicial complex, the pair $(|K|,|L|)$ of the geometric realizations has the (topological) homotopy extension property. We want to define a purely combinatorial analogue for a simplicial $\operatorname{pair}(K, L)$. 
Definition 7.1. A simplicial map $i: L \rightarrow K$ has the contiguity extension property if given two simplicial maps $\varphi, \psi: L \rightarrow M$ which lie in the same contiguity class, $\varphi \sim \psi$, and given an extension of $\varphi$ (that is, a simplicial map $\tilde{\varphi}: K \rightarrow M$ such that $\tilde{\varphi} \circ i=\varphi$ ), there exists an extension $\tilde{\psi}$ of $\psi$ with $\tilde{\varphi} \sim \tilde{\psi}$ :

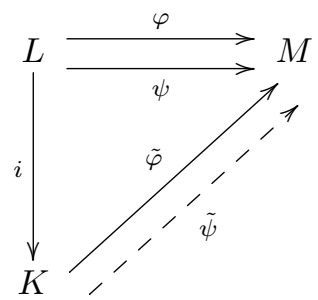

Example 7.2. Let $K_{0}$ be the core of the complex $K$. Then the inclusion $i: K_{0} \subset K$ is a simplicial cofibration. In fact, as explained before, there is a simplicial retraction $r: K \rightarrow K_{0}$ such that $r \circ i=1_{K_{0}}$ and $i \circ r \sim 1_{K}$. Then one can take $\tilde{\psi}=r \circ \psi$ because $\tilde{\varphi} \sim \varphi \circ r$.

As an application of Theorem 6.10, we shall show that there are simplicial pairs $(K, L)$ that do not have the extension property.

Theorem 7.3. Let $K$ be a connected simplicial complex with scat $K=$ $n$. Assume that there is a categorical covering $L_{1}, \ldots, L_{n+1}$ of $K$ such that all the pairs $\left(K, L_{j}\right)$ have the contiguity extension property. Then scat $^{\mathrm{Wh}} K=n$.

Proof: Due to Theorem 6.6 we only have to prove that scat ${ }^{\mathrm{Wh}} K \leq n$. By hypothesis, each inclusion $I_{j}: L_{j} \rightarrow K$ is in the same contiguity class that some constant map, whose image is a vertex $v_{j}$. Since two such constant maps are contiguous if and only if the vertices $v_{i}, v_{j}$ lie on the same simplex, and $K$ is connected, we can suppose that all the vertices are equal, say to some base point $v_{0}$. Taking $i=I_{j}, \varphi=I_{j}, \psi=v_{0}$, and $\tilde{\varphi}=1_{K}$, the simplicial extension property gives maps $\tilde{\psi}_{j} \sim 1_{K}$ such that $\tilde{\psi}_{j}\left(L_{j}\right)=\left\{v_{0}\right\}$. Define the map

$$
\delta: K \rightarrow T^{n+1} K, \quad \delta(v)=\left(\tilde{\psi}_{1}(v), \ldots, \tilde{\psi}_{n+1}(v)\right) .
$$

It is well defined, because each vertex $v$ is contained in some $L_{j}$, hence $\tilde{\psi}_{j}(v)=v_{0}$, meaning that the $j$-th coordinate of $\delta(v)$ is the base point. Moreover $\delta$ is a simplicial map. In fact, for any simplex $\sigma \in K$ we have that $\delta(\sigma)$ is a simplex in $K^{n}$ (Definition 5.1). Moreover, $\sigma$ must be contained in some $L_{j}$, and $\delta\left(L_{j}\right) \subset K_{j}$. Then $\delta(\sigma) \in K_{j} \subset T^{n+1} K$. Now, let

$$
\tilde{\psi}_{j}=\varphi_{j}^{1} \sim_{c} \cdots \sim_{c} \varphi_{j}^{m}=1_{K}
$$


be the sequence of contiguous maps connecting $\tilde{\psi}_{j}$ and $1_{K}$ (clearly we may assume that the length $m$ does not depend on $j$ ). Then the maps

$$
\left(\tilde{\varphi}_{1}^{k}, \ldots, \tilde{\varphi}_{n+1}^{k}\right): K \rightarrow K^{n+1}, \quad k=1, \ldots, m,
$$

define a sequence of contiguous maps between $J \circ \delta=\left(\tilde{\psi}_{1}, \ldots, \tilde{\psi}_{n+1}\right)$ and $\Delta=(1, \ldots, 1)$. Hence $J \circ \delta \sim \Delta$, which means that scat $^{\mathrm{Wh}} K \leq n$.

Remark 7.4. It is interesting to point out that from Theorem 7.3 and the proof of Theorem 6.6 it follows that scat $K=$ scat $^{\text {Wh }} K$ if and only if there is a categorical covering $L_{1}, \ldots, L_{n+1}$ where each pair $\left(K, L_{j}\right)$ has the contiguity extension property.

As a corollary, some categorical covering of the complex $K$ in Figure 6 fails to verify the hypothesis of the preceding theorem, because scat $K=1$ but scat ${ }^{\mathrm{Wh}} K>2$.

Example 7.5. The subcomplex $L=K \backslash \sigma$, of the complex $K$ in Figure 6 , where $\sigma=\{a, b, c\}$, has not the contiguity extension property. Let $v$ be the upper vertex of $K$ (see Figure 6 ). Since $L$ is strongly collapsible to $v$, the inclusion $\varphi=i: L \rightarrow K$ and the constant map $\psi=c_{v}: L \rightarrow K$ lie in the same contiguity class. Fix $\tilde{\varphi}=1_{K}: K \rightarrow K$ to be the identity. If there exists $\tilde{\psi} \sim 1_{K}$ such that $\psi(L)=\{v\}$ there must be some sequence $\tilde{\psi} \sim_{c} \varphi_{1} \sim_{c} \cdots \sim_{c} 1_{K}$ of contiguous maps. However, since $a, b, c \in L$, the simplicial map $\varphi_{1}$ must be constant, and we obtain that $1_{K}$ is contiguous to a constant map, which is a contradiction because scat $K \neq 0$.

Example 7.6. A simpler example was communicated to us by N. Scoville: let $K$ be the 1-dimensional complex in Figure 7, with vertices $a$, $b, c$ and let $\sigma$ be the 1-simplex joining the vertices $b$ and $c$. Then the subcomplex $L=K \backslash \sigma$ has not the contiguity extension property.

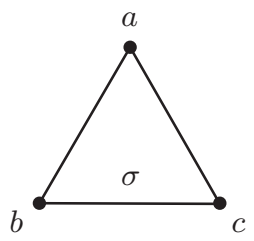

Figure 7. The subcomplex $L=K \backslash \sigma$ has not the contiguity extension property. 


\section{Graphs}

8.1. Arboricity. This section is focused on the study of the simplicial LS-category in the one-dimensional case, that is, on graphs. The well known graph-theoretical notion of arboricity will play a central role in this study. Basically, arboricity is based on the cardinality of minimal decompositions of a graph into disjoint spanning forests, i.e., acyclic subgraphs, which are non-necessarily connected and cover all the vertices.

The aim of this section is to prove that arboricity coincides (up to one) with both simplicial and geometric simplicial categories.

Remark 8.1. In [1], Aaronson and Scoville introduced a so-called discrete LS-category in the simplicial setting. They proved that, for the 1-dimensional case, it is equivalent to arboricity.

Let us start with some basic notions on graph theory. A general reference is Harary's book [12].

Definition 8.2. Let $G$ be a graph. A cycle in $G$ is an alternating sequence of distinct vertices and edges, $v_{0}, e_{1}, v_{1}, \ldots, v_{n-1}, e_{n}, v_{n}$, where the incident vertices of each edge $e_{i}$ are $v_{i-1}$ and $v_{i}$ respectively, and such that $v_{0}=v_{n}$.

Under a topological point of view, cycles are triangulations of the circle $S^{1}$.

Definition 8.3. A forest is a graph without cycles, alternatively it can be called acyclic graph. A tree is a connected forest.

Definition 8.4. The arboricity of a graph $G$, denoted by $\Upsilon(G)$, is the minimum number of edge-disjoint spanning forests into which $G$ can be decomposed.

Nash-Williams [19] determined the arboricity of a general graph:

Theorem 8.5 ([12, Theorem 9.10]). Let $G$ be a nontrivial graph and let $q_{n}$ be the maximum number of edges in any subgraph of $G$ with $n$ vertices. Then

$$
\Upsilon(G)=\max _{n}\left\lceil\frac{q_{n}}{n-1}\right\rceil .
$$

Example 8.6. For the particular case of complete graphs (see Figure 4) it follows the following formula: $\Upsilon\left(K_{2 n}\right)=n=\Upsilon\left(K_{2 n-1}\right)$.

8.2. Categorical subgraphs and forests. Now we prove some results which will be used later in this section. 
Remark 8.7. Every standard elementary collapse in a graph is the deletion of a so-called leaf vertex $v$ and the unique edge $v v^{\prime}$ containing $v$. Thus $v$ is dominated by $v^{\prime}$ and hence, in graphs, every elementary collapse is an elementary strong collapse.

Lemma 8.8. Let $G$ be a connected graph and let $L \subset G$ be a subgraph containing at least one cycle $C$. If $\varphi: L \rightarrow G$ is a simplicial map contiguous to the inclusion $i_{L}: L \rightarrow G$, then $\varphi(L)$ contains the cycle $C$. Moreover $\varphi(L) \subset L$ and $\varphi_{\mid C}$ is the inclusion $i_{C}: C \rightarrow G$.

Proof: Every edge $e$ in $L$ satisfies that $\varphi(e) \cup e$ is a simplex in $G$ (for graphs, "simplex" means a vertex or an edge), so, equivalently $\varphi(e) \subseteq e$; therefore, either $\varphi(e)=e$ or $\varphi(e)$ is one of the extreme vertices of $e$. This implies that $\varphi(L) \subseteq L$.

Let $C$ be a cycle contained in $L$ and let us consider the restrictions to $C$ of $\varphi$ and the inclusion $i_{L}$, denoted by $\varphi_{\left.\right|_{C}}$ and $i_{C}$ respectively, which are also contiguous as consequence of the fact that the composition of contiguous maps is contiguous. So, if $\varphi$ maps every edge of the cycle $C$ onto itself then $\varphi(C)=C$ and hence $\varphi(L)$ contains the cycle $C$. Otherwise, there exists an edge $e_{1}$ in $C$ such that $\varphi\left(e_{1}\right) \neq e_{1}$; we can suppose without loss of generality that $\varphi\left(e_{1}\right)=v_{0}$. Now let us consider the edge $e_{2}$ which is adjacent to $e_{1}$ in $v_{1} \neq v_{0}$. Since the map $\varphi$ is simplicial, we have $\varphi\left(v_{1}\right)=v_{0}$, which is a contradiction with $\varphi\left(e_{2}\right) \subseteq e_{2}$. Finally, we conclude that all the edges in $C$ remain fixed by $\varphi$ so $\varphi(L)$ contains at least one cycle.

Remark 8.9. Let $P \subset G$ be a a path in a graph, such that there is a simplicial map $\varphi: P \rightarrow G$ contiguous to the inclusion $i_{P}: P \rightarrow G$. By the same argument of the proof above, any edge $e$ contained in $P$ satisfies that either $\varphi(e)=e$ or $\varphi(e)=u$, where $u$ is one of the extreme vertices of $e$ with degree 1 , i.e. $u$ is a so-called leaf vertex of $P$. Thus, we conclude that the only possible reductions induced by a simplicial map contiguous to the inclusion are given by standard collapses.

The next result establishes the equivalence for graphs between the notions of categorical subcomplex and acyclic subgraph.

Theorem 8.10. Let $G$ be a connected graph and let $L \subseteq G$ be a subgraph. Then $L$ is categorical in $G$ if and only if $L$ is a forest.

Proof: Let us suppose that there exists a categorical but non-acyclic subgraph $L \subset G$. By definition, there exists a vertex $v \in G$ such that the inclusion $i=i_{L}: L \rightarrow G$ and the constant map $c=c_{v}: L \rightarrow G$ are in the 
same contiguity class, which gives a sequence $i_{L}=\varphi_{1} \sim_{c} \cdots \sim_{c} \varphi_{m}=c_{v}$ of directly contiguous maps $\varphi_{i}: L \rightarrow G$.

Now, since $i_{L} \sim_{c} \varphi_{2}$ and $L$ contains at least one cycle $C$, by taking into account the previous lemma we conclude that $\varphi_{2}(L)$ contains at least the cycle $C$. Moreover, $\left(\varphi_{2}\right)_{\mid C}=i_{C}$ is the inclusion $i_{C}: C \subset G$. Now, $\varphi_{2} \sim_{c} \varphi_{3}$ implies, by composing with the inclusion $\varphi_{2}(L) \subset L$, that $i_{C}=\left(\varphi_{2}\right)_{\mid C} \sim_{c}\left(\varphi_{3}\right)_{\mid C}$. That means, by applying the lemma again, that $\varphi_{3}(C)$ is the cycle $C$, and by repeating the argument we shall arrive to a cycle that can be deformed into a point, which is impossible. Hence we conclude that $L$ is an acyclic subgraph of $G$, that is, $L$ is a forest.

Conversely, let us assume that $L$ is a forest, so $L$ is a disjoint union of trees $T_{i}$ with $i=1, \ldots, n$. It is clear that each inclusion map $T_{i} \subset G$ is in the same contiguity class as the constant map sending the tree $T_{i}$ onto one of its vertices $v_{i}$. Since $G$ is connected, there is at least one path in $G$ joining every vertex $v_{i}$ with a given vertex $v_{0}$. Then the inclusion of $L$ is in the same contiguity class as the constant map $v$. Hence $L$ is a categorical subcomplex in $G$.

Lemma 8.11. Let $G$ be a connected graph. For every covering of $G$ by forests there exists a covering of $G$ by trees with the same number of elements.

Proof: Let $F_{1}, \ldots, F_{k}$ be a covering of $G$ by forests. Since $G$ is connected, it follows that given two trees $T$ and $T^{\prime}$ in a forest $F_{i}$ and any vertices $v \in T$ and $v^{\prime} \in T^{\prime}$, they can be linked by means of a path in $G$. By adding such a path to $F_{i}$, and removing, if necessary, the edges of the path which create cycles and are not contained in $F_{i}$, we link the trees $T$ and $T^{\prime}$ of $F_{i}$ (see Figure 8).

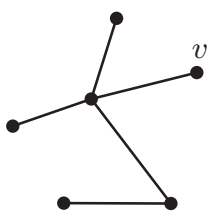

$F_{i}$
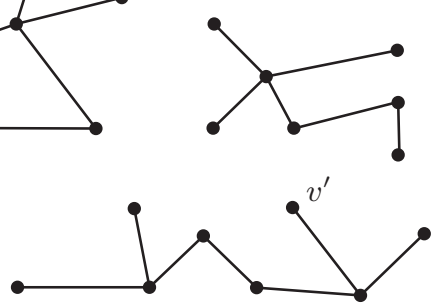

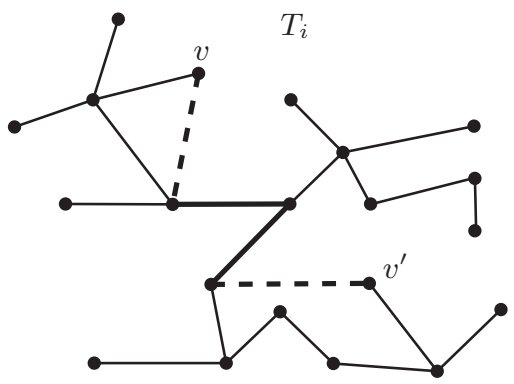

Figure 8. Converting a forest into a tree. 
Repeating the argument for the remaining trees, we obtain one tree $T_{i}$ containing $F_{i}$. Finally, carrying out the same procedure on every forest of $G$, we obtain a covering of $G$ by trees $T_{1}, \ldots, T_{k}$.

8.3. Main result. We now state the main result of this section.

Theorem 8.12. Let $G$ be a connected graph. Then gscat $G=\Upsilon(G)-1$.

Proof: Let us suppose that $\Upsilon(G)=k+1$, so by definition there exists a covering of $G$ with $k+1$ edge-disjoint spanning forests. By means of Lemma 8.11 we can construct a covering of $G$ with $k+1$ trees. Since the trees are strongly collapsible we conclude that gscat $G \leq k$.

Conversely, let us assume that gscat $G=k$. It means that there is a covering of $G$ with $k+1$ strongly collapsible subsomplexes. By Theorem 8.10, these complexes are trees $T_{0}, \ldots, T_{k}$. Starting from a tree $T_{i}$, we can obtain a spanning forest $F_{i}$ by adding all the isolated vertices which are not covered by $T_{i}$. Next, if an edge is contained in several forests, in order to obtain a covering by edge-disjoint forests, we remove it from all these forests but one. Hence $\Upsilon(G) \leq k+1$.

Corollary 8.13. For any graph $G$, we have scat $G=\operatorname{gscat} G=\Upsilon(G)-1$.

Proof: Apply again Lemma 8.8 and Theorem 8.10 separately on each connected component of $G$.

In Section 3, the behaviour of scat under barycentric subdivisions was studied. Actually, it was proved that $\operatorname{scat}(\operatorname{sd} K) \leq \operatorname{scat} K$. In the one-dimensional case there are examples where this inequality is strict (see Example 3.6). Also there are examples of 2-dimensional complexes where $\operatorname{scat}\left(\mathrm{sd}^{N} K\right)$ remains constant for all $N$ and is strictly greater than the topological LS-category of the geometric realization cat $|K|$ (see Example 3.6).

In contrast, in the one-dimensional case it holds the geometric category of the first barycentric subdivision always reaches the topological category of the geometric realization. In fact, the following result states that this is possible for a certain kind of "local" barycentric subdivision.

Remember that any finite CW complex $X$ satisfies cat $X \leq \operatorname{dim} X$, therefore cat $|K|=1$ for a non-contractible graph.

Proposition 8.14. Let $G$ be a connected graph. If $G$ is a tree then scat $G=$ cat $|G|=0$. Otherwise, let $G^{\prime}$ be the subdivision obtained from $G$ by only bisecting those edges out of a spanning tree in $G$. Then scat $G^{\prime}=\operatorname{cat}|G|=1$. 
Proof: Let us consider a spanning tree $T$ in $G$. It is well known $[\mathbf{1 3}$, Proposition 1A.2] that there is a bijection between the edges out of a spanning tree and the basic cycles generating the one-dimensional homology of a graph. Now, after every edge out of $T$ is barycentrically subdivided, the subdivision $G^{\prime}$ of $G$ can be covered by two different (non disjoint) spanning trees $T_{1}$ and $T_{2}$ constructed as follows (see Figure 5): $T_{1}$ is an expansion of $T$ which is obtained by adding one edge (one of the subdivided ones) on every leaf vertex of $T$; analogously, $T_{2}$ is obtained by adding the other edge (not previously added to construct $T_{1}$ ) to all the leaf vertices of $T$. By definition both $T_{1}$ and $T_{2}$ are spanning trees covering $G^{\prime}$ and hence scat $G^{\prime}=$ cat $|G|=1$.

Example 8.15. Notice that for certain graphs, as the complete ones $K_{n}$, the simplicial LS-category equals the topological LS-category of the realization of the graph by bisecting a fewer number of edges than the stated one by the above proposition. For example, for $K_{5}$ only three edges must be bisected in order to get scat $K_{5}^{\prime}=1$ (see Figure 9).

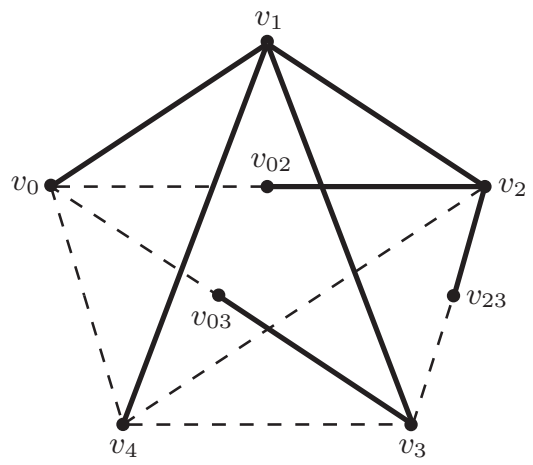

Figure 9. Bisection of three edges to obtain the category of the geometric realization.

Corollary 8.16. Let $G$ be a connected graph. Then $\operatorname{scat}(\operatorname{sd} G)=$ cat $|G|$.

Remark 8.17. Taking into account the above results, it is interesting to point out that, in the one-dimensional case, the difference scat $G-$ scat $(\operatorname{sd} G)=\operatorname{scat} G-$ cat $|G|$ can be arbitrarily large, as can be checked by considering the complete graph $K_{n}$. 
Acknowledgements. We thank J. Barmak for his valuable suggestions and M. J. Pereira-Sáez for many useful discussions. We are grateful to J. Oprea for pointing out reference $[\mathbf{1 4}]$ to us and R. D. Helmstutler for some explanations about his paper.

\section{References}

[1] S. Aaronson and N. A. Scoville, Lusternik-Schnirelmann category for simplicial complexes, Illinois J. Math. 57(3) (2013), 743-753.

[2] R. Ayala, E. Domínguez, And A. Quintero, Topología poliedral, Publicaciones del Seminario Matemático "García de Galdeano", Serie 2, Sección 3, 12 (1987).

[3] J. A. BARMAK, "Algebraic Topology of Finite Topological Spaces and Applications", Lecture Notes in Mathematics 2032, Springer, Heidelberg, 2011. DOI: 10.1007/978-3-642-22003-6.

[4] J. A. Barmak and E. G. Minian, Strong homotopy types, nerves and collapses, Discrete Comput. Geom. 47(2) (2012), 301-328. DOI: 10.1007/s00454-0119357-5.

[5] M. M. Cohen, "A Course in Simple-Homotopy Theory", Graduate Texts in Mathematics 10, Springer-Verlag, New York-Berlin, 1973. DOI : 10.1007/978-14684-9372-6.

[6] O. Cornea, G. Lupton, J. Oprea, and D. Tanré, "Lusternik-Schnirelmann Category", Mathematical Surveys and Monographs 103, American Mathematical Society, Providence, RI, 2003. DOI : 10.1090/surv/103.

[7] A. Dold, "Lectures on Algebraic Topology", Die Grundlehren der mathematischen Wissenschaften 200, Springer-Verlag, New York-Berlin, 1972. DOI: 10.1007/978-3-662-00756-3.

[8] S. Eilenberg and N. Steenrod, "Foundations of Algebraic Topology", Princeton University Press, Princeton, New Jersey, 1952.

[9] D. Fernández-Ternero, E. Macías-Virgós, and J. A. Vilches, LusternikSchnirelmann category of simplicial complexes and finite spaces, Topology Appl. 194 (2015), 37-50. DOI : 10.1016/j.topol.2015.08.001.

[10] R. H. Fox, On the Lusternik-Schnirelmann category, Ann. of Math. (2) 42(2) (1941). 333-370. DOI : 10.2307/1968905.

[11] R. Fritsch and R. A. Piccinini, "Cellular Structures in Topology", Cambridge Studies in Advanced Mathematics 19, Cambridge University Press, Cambridge, 1990. DOI : $10.1017 /$ CB09780511983948.

[12] F. Harary, "Graph Theory", Addison-Wesley Publishing Co., Reading, Mass.Menlo Park, Calif.-London 1969.

[13] A. Hatcher, "Algebraic Topology", Cambridge University Press, Cambridge, 2002.

[14] R. D. Helmstutler and R. M. Vaughn, Finite co-H-spaces are contractible: A dual to a theorem of Stong, Preprint (2012), available online at: http://files. umwblogs .org/blogs.dir/4710/files/2010/10/RDH-RMV-co-H.pdf.

[15] I. M. James (ED.), "History of Topology", North-Holland, Amsterdam, 1999.

[16] D. KozLov, "Combinatorial Algebraic Topology", Algorithms and Computation in Mathematics 21, Springer, Berlin, 2008. DOI : 10.1007/978-3-540-71962-5. 
[17] A. T. Lundell and S. Weingram, "The topology of CW Complexes", The University series in higher mathematics, Van Nostrand Reinhold Co., New York, 1969. DOI : $10.1007 / 978-1-4684-6254-8$.

[18] M. C. MCCord, Singular homology groups and homotopy groups of finite topological spaces, Duke Math. J. 33(3) (1966), 465-474. DOI : 10.1215/S0012-709466-03352-7.

[19] C. St. J. A. Nash-Williams, Edge-disjoint spanning trees of finite graphs, $J$. London Math. Soc. 36(1) (1961), 445-450. DOI : 10.1112/j1ms/s1-36.1.445.

[20] E. H. Spanier, "Algebraic Topology", Springer-Verlag, New York, 1966. DOI: 10.1007/978-1-4684-9322-1.

Desamparados Fernández-Ternero

José Antonio Vilches

Departamento de Geometría y Topología, Universidad de Sevilla, Spain

E-mail address: desamfer@us.es

E-mail address: vilches@us.es

Enrique Macías-Virgós

Departamento de Matemáticas, Universidade de Santiago de Compostela, Spain

E-mail address: quique.macias@usc.es

Erica Minuz

Department of Mathematics, Ny Munkegade 118, Building 1530, 333, 8000 Aarhus C, Denmark

E-mail address: minuz@math.au.dk

Primera versió rebuda el 25 de maig de 2017, darrera versió rebuda el 24 d'octubre de 2017. 